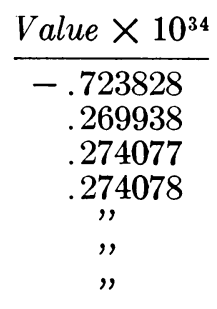

\begin{tabular}{ccc}
\multicolumn{3}{c}{ Evaluation of $K_{i 50}(20)$} \\
\hline$v$ & $\frac{B}{.0025}$ \\
-1.00 & 3.65 &,$"$ \\
-1.20 & 4.00 & $"$, \\
-1.40 & 4.80 & .005 \\
-1.58 & 5.45 & $"$, \\
-1.52 & 5.60 &
\end{tabular}

Acknowledgement. This work was supported by the Atomic Energy Commission under Contract No. A'T(30-1)-2772 which included access to the CDC-6600 facility at the Courant Institute for Mathematical Sciences at New York University.

Lehigh University

Bethlehem, Pennsylvania

E. I. D॥ Pont Nemours

Wilmington, Delaware 19898

1. J. M. JACKson \& N. F. Motr, "Energy exchange between inert gas atoms and a solid surface," Proc. Roy. Soc. London Ser. A, v. 137, 1932, pp. 703-717.

\title{
Mixed Algebraic-Exponential Interpolation Using Finite Differences
}

\author{
By J. W. Layman
}

The use of finite differences in exponential polynomial interpolation was introduced in [1], where an algorithm was developed which triangularizes the system of cquations that determines the coefficients in the interpolating exponential polynomial. In the present note we show that a similar finite-difference algorithm also exists for interpolation by a mixed algebraic-exponential polynomial of the form

$$
P(x)=\sum_{n=1}^{N} \sum_{m=0}^{m_{n}} a_{n m} x^{(m)} n^{x}
$$

for $x=0,1,2, \cdots, \sum_{n=1}^{I}\left(m_{n}+1\right)-1$. The symbol $x^{(m)}$ represents the factorial power function $x(x-1) \cdots(x-m+1)$.

We require the basic difference operations $E$ and $\Delta$ and, in addition, the diagonal difference $S$ defined by $S f(x)=\Delta^{x} f(0)$. The diagonal difference is more precisely defined in [1] and certain difficulties in interpretation are resolved there. These arise when taking higher-order diagonal differences by iteration, $S^{n} f(x)=S S^{n-1} f(x)$.

The following properties and formulas involving the diagonal-difference opera-

Received April 28, 1966. Revised December 5, 1966. 
tion follow more or less directly from the definition. Proofs are left to the reader.

$$
\begin{aligned}
S[a f(x)+b g(x)] & =a S f(x)+b S g(x), \\
S^{m} f(x) & =(E-m)^{x} f(0), \\
S^{n}\left[x^{(r)} f(x)\right] & =x^{(n)} E^{-r} S^{n} f(k+r), \\
S^{m}\left[n^{x} f(x)\right] & =n^{x} S^{m / n} f(x), \text { if } n \text { divides } m, \\
S\left[x^{(r)} a^{x}\right] & =x^{(r)} a^{r}(a-1)^{x-r} .
\end{aligned}
$$

For consistency we define $x^{(r)} 0^{x-r}$ to be zero for $x<r, r$ ! for $x=r$, zero for $x>r$.

Rather than developing the triangularization procedure for the general algebraic-exponential polynomial in (1), we will restrict ourselves to the special case of $N=2$ with $m_{1}=m_{2}=2$. Then we may write

$$
P(x)=a_{1}+b_{1} x+c_{1} x^{(2)}+\left(a_{2}+b_{2} x+c_{2} x^{(2)}\right) 2^{x} .
$$

We now apply the operators $E$ and $S$ in the appropriate sequence so that the coefficients are eliminated one-by-one from left to right to obtain the following:

$$
\begin{array}{rrr}
f(x) & =a_{1}+b_{1} x+c_{1} x^{(2)}+\left(a_{2}+b_{2} x+c_{2} x^{(2)}\right) 2^{x}, \\
S f(x) & =a_{1} 0^{x}+b_{1} x 0^{x-1}+c_{1} x^{(2)} 0^{n-2}+a_{2}+2 b_{2} x+2^{2} c_{2} x^{(2)}, \\
E S f(x) & = & b_{1}(x+1) 0^{x}+c_{1}(x+1)^{(2)} 0^{x-1}+2 b_{2}(x+1)+4 c_{2}(x+1)^{(2)}, \\
E^{2} S f(x) & = & c_{1}(x+2)^{(2)} 0^{x}+2 b_{2}(x+2)+4 c_{2}(x+2)^{(2)}, \\
E^{3} S f(x) & = & a_{2}+2 b_{2}(x+3)+4 c_{2}\left[x^{(2)}+8 x+12\right], \\
S E^{3} S f(x) & a_{2} 0^{x}+2 b_{2} x 0^{x-1}+6 b_{2} 0^{x}+4 c_{2}\left[(x+1)^{(2)}\right. \\
& & \left.+6 x 0^{x-1}+6 \cdot 0^{x}\right], \\
E S E^{3} S f(x) & = & 2 b_{2}(x+1) 0^{x}+4 c_{2}\left[(x+1)^{(2)} 0^{x-1}\right. \\
E^{2} S E^{3} S f(x) & & \left.+6(x+1) 0^{x}\right], \\
& 4 c_{2}(x+2)^{(2)} 0^{x} .
\end{array}
$$

This system is triangular for any $x$; however, the smallest number of data points is required if we take $x=0$. Several redundant equations can be eliminated to yield the following system:

$$
\begin{array}{rlrl}
S f(0) & =a_{1} & +a_{2}, \\
E S f(0) & = & b_{1} & +a_{2}+2 b_{2}, \\
E^{2} S f(0) & = & 2 c_{1}+a_{2}+4 b_{2}+8 c_{2}, \\
S E^{3} S f(0) & = & a_{2}+6 b_{2}+24 c_{2}, \\
E S E^{3} S f(0) & = & 2 b_{2}+24 c_{2}, \\
E^{2} S E^{3} S f(0) & = & 8 c_{2} .
\end{array}
$$

For any given instance of the general algebraic-exponential polynomial we proceed in a similar manner. We first apply the operator $S$, followed by $n+1$ successive applications of $E$, where $n$ is the degree of $A_{1}(x)$, then again apply $S$, followed by $m+1$ successive applications of $E$ where $m$ is the degree of $A_{2}(x), \cdots$ etc. We evaluate these derived polynomials at $x=0$ and discard redundant equations to obtain the appropriate triangularization scheme.

Consideration of the diagonal-difference operation $S$ shows that $S f(x)$ is simply the diagonal of so-called leading differences, i.e., the diagonal of differences which passes through $f(0)$. The function $S E^{3} S f(x)$, for example, is then obtained by calculating the diagonal of leading differences of $E^{3} S f(x)$, that is, the diagonal of differences of $S f(x)$ through $S f(3)$. A typical example is shown below. 


\begin{tabular}{|c|c|c|c|c|c|c|c|c|c|}
\hline$x$ & $f(x)$ & & & & & & $\operatorname{Sf}(x)$ & & \\
\hline 0 & 1 & & & & & & 1 & & $1=S f(0)$ \\
\hline 1 & -1 & -2 & & & & & -2 & & $-2=\operatorname{ES} S f(0)$ \\
\hline 2 & 2 & 3 & 5 & & & & 5 & & $5=E^{2} S f(0)$ \\
\hline 3 & 1 & -1 & -4 & -9 & & & -9 & & $-9=S E^{3} S f(0)$ \\
\hline 4 & -8 & -9 & -8 & -4 & 5 & & 5 & 14 & $14=E S E^{3} S f(0)$ \\
\hline 5 & 3 & 11 & 20 & 28 & 32 & 27 & 27 & 22 & $8=E^{2} S E^{3} S f(0)$ \\
\hline
\end{tabular}

Substituting the values of $S f(0), \cdots, E^{2} S E^{3} S f(0)$ from the tabulation into (3) yields a triangular system which is easily solved to obtain:

$$
\begin{array}{lll}
a_{1}=4, & b_{1}=11, & c_{1}=10, \\
a_{2}=-3, & b_{2}=-5, & c_{2}=1 .
\end{array}
$$

Hence the algebraic-exponential polynomial of the form given in Eq. (2) which fits the given data is

$$
P(x)=4+11 x+10 x^{(2)}+\left[-3-5 x+x^{(2)}\right] 2^{x} .
$$

The principal advantage of the present method is just that of any finite-difference interpolation method, that is, the systematic handling of the given data. For example, Gauss elimination can be used to give, as the fifth equation of (3),

$$
2 b_{2}+24 c_{2}=f_{4}-5 f_{3}+9 f_{2}-7 f_{1}+2 f_{0},
$$

which the present method gives as $2 b_{2}+24 c_{2}=E S E^{3} S f_{0}$, a result which clearly indicates a systematic difference-table computation procedure. Furthermore, an analysis of the numerical example shows that Gauss elimination requires 15 additions (subtractions) and 12 multiplications involving the given data $f_{0}, f_{1}, \cdots, f_{5}$, to triangularize the coefficient matrix, whereas the present method requires 18 subtractions.

It may be pointed out that the present method leads to a strictly diagonal system when no exponential factors $a^{x}$ appear, equivalent to the Gregory-Newton lorward Interpolation method. When no algebraic factors appear we obtain the exponential polynomial method of [1].

For a remainder analysis, when approximating functions of known properties, see a paper by Gori [2].

Virginia Polytechnic Institute

Blacksburg, Virginia 24061

1. J. W. Layman, "A finite-difference exponential approximation method," Math. Comp., v. 18,1964, pp. 113-118.

2. LAURA GorI, "Una generalizzazione della formula di interpolazione di Lagrange-Hermite," Ricerche Mat., v. 9, 1960, pp. 242-247. MR 27 \#1758. 